\title{
Estudio de la intercara de una preforma híbrida infiltrada sin presión
}

\author{
M. Merchán*, M. García de Cortázar*, P. Egizabal*, F. Peñalba*, A. J. López**, B. Torres**, P. Rodrigo** y J. Rams** \\ Resumen \\ En este trabajo se presenta la caracterización de la intercara matriz/refuerzo en un compuesto de matriz de aluminio \\ reforzado con fibras de Saffil y partículas de alúmina, fabricado mediante el proceso de infiltración sin presión, \\ Primex $^{\mathrm{TM}}$. La caracterización microestructural y química del material compuesto se ha realizado mediante micros- \\ copía electrónica de barrido y mediante análisis semicuantitativo por energía dispersiva de rayos-X. Asimismo, se \\ ha realizado una caracterización mecánica del material compuesto utilizando la técnica de nanoindentación. De la \\ investigación se concluye que se tiene una buena unión entre los refuerzos y la matriz.
}

Palabras clave

Infiltración sin presión; Intercara; Preforma; Nanoindentación.

\section{Study of the interface of a pressureless infiltrated hybrid preform}

\begin{abstract}
This work presents an interfacial characterization of the matrix/reinforcement interface of an aluminium matrix composite reinforced with Saffil fibres and alumina particles was manufactured using the Primex ${ }^{\mathrm{TM}}$ pressureless infiltration process. Scanning electron microscopy and energy dispersive X-ray spectroscopy were used to carry out the microstructural and chemical characterization of the composite material. In addition, a mechanical characterization was conducted by the nanoindentation technique. This research concludes that the composite presents a good bonding between the matrix and the reinforcements.
\end{abstract}

Keywords

Pressureless infiltration; Interface; Preform; Nanonindentation.

\section{INTRODUCCIÓN}

Los materiales compuestos de matriz de aluminio (AMCs) se presentan como alternativa interesante en numerosas aplicaciones, debido a su alta rigidez y resistencia específica, elevada resistencia al desgaste, alta conductividad térmica y bajo coeficiente de expansión térmica. Se trata de materiales que están siendo reconsiderados en aplicaciones de transporte, debido al potencial que presentan para reducir el peso e incrementar la eficiencia. Sin embargo, los complejos procesos de producción empleados para su fabricación y el coste siguen constituyendo algunas limitaciones clave para la expansión del uso de los AMCs, en una industria tan sensible al precio como es la de automoción.

El método de fabricación comúnmente utilizado para la obtención de AMCs ha sido el de la infiltración de preformas cerámicas por presión. La infiltración de aluminio fundido en el interior de preformas cerámicas porosas reduce los defectos derivados de la absorción de aire que se manifiestan, habitualmente, en procesos de fundición como en el stir casting o moldeo por agitación. Sin embargo, este efecto se compensa de forma negativa por la aparición de otros defectos, como macrosegregaciones y defectos de contracción, como consecuencia de las altas presiones que se requieren para introducir el metal fundido en la preforma. El proceso de infiltración sin presión PRIMEX ${ }^{\mathrm{TM}}$, desarrollado por la Corporación Lanxide, es un proceso alternativo para fabricar materiales compuestos a través de la infiltración de cuerpos porosos con aleaciones metálicas ligeras. El cuerpo poroso puede estar en forma de una cama de polvo suelto o de una preforma de material de refuerzo. A diferencia de la infiltración asistida por presión, el proceso PRIMEX ${ }^{\mathrm{TM}}$ permite que la infiltración ocurra de forma espontánea, sin la necesidad de aplicar presión externa.

El proceso de infiltración sin presión ha centrado la atención de numerosos investigadores en los

\footnotetext{
* INASMET-Tecnalia Paseo de Mikeletegi, 2, 20009 San Sebastián.

** Dpto. de Ciencia e Ingeniería de Materiales, Universidad Rey Juan Carlos, C/ Tulipán s/n, 28933 Móstoles, Madrid.
} 
últimos años, debido al potencial que presenta para la obtención de $\mathrm{AMC}$ con altos niveles de refuerzo y la posibilidad de fabricar componentes con forma cercana a la final. Un aspecto, este último, muy interesante si se quieren fabricar componentes de AMC a un precio competitivo, ya que se reducen las tan costosas operaciones de acabado de este tipo de materiales. Las principales investigaciones realizadas para la evaluación de este proceso han estado centradas en preformas compuestas de $\mathrm{SiC}_{\text {y }} \mathrm{Al}_{2} \mathrm{O}_{3}{ }^{[1 \text { y } 2]}$. Las preformas de fibras cortas también han sido infiltradas aunque, para ello, de momento, se siguen usando métodos de infiltración asistidos por presión o en vacío ${ }^{[3]}$. La orientación de las fibras en un plano facilita la infiltración de las preformas de fibras en comparación con las preformas de partículas, en las que éstas tienen tendencia a unirse y crear estructuras que reducen el espacio entre ellas. Sin embargo, la tendencia de las fibras a recuperar su forma, tras la aplicación de presión durante el proceso de fabricación de las preformas, limita las posibilidades de diseño de éstas, reduciendo mucho los campos de aplicación de los componentes fabricados. Las preformas híbridas, compuestas por fibras y partículas dispuestas de forma homogénea, pueden suponer una interesante alternativa ya que se benefician de las ventajas de ambos tipos de refuerzos. Se pueden conseguir elevados porcentajes de refuerzo y, además, preformas con formas complejas. Otra de las ventajas es el coste de este tipo de preformas, ya que se pueden sustituir las fibras por partículas más económicas.

La intercara matriz/refuerzo es la superficie de unión a través de la cual se crea una discontinuidad en la composición química, módulo elástico, coeficiente de expansión térmica y propiedades termodinámicas. La importancia de la intercara en los materiales compuestos radica en que determina las propiedades finales del material. La intercara ideal de un compuesto de matriz metálica debería promover la mojabilidad y la unión del refuerzo con la matriz hasta el grado deseado. Las reacciones interfaciales en las preformas de partículas infiltradas sin presión por aleaciones de aluminio han sido estudiadas con objeto de poder entender mejor el mecanismo de infiltración en este proceso ${ }^{[1-4]}$. Sin embargo, las intercaras creadas en la infiltración sin presión de preformas de fibras lo han sido menos y son objeto de estudio, actualmente.

Para el estudio de dichas intercaras, en la presente investigación, se han fabricado probetas de material compuesto empleando el proceso PRIMEX ${ }^{\mathrm{TM}}$, donde se han infiltrado preformas híbridas compuestas por fibras Saffil y partículas de alúmina con una aleación de aluminio. La caracterización microestructural y química del material compuesto se ha realizado mediante microscopía electrónica de barrido (SEM) y mediante análisis semicuantitativo, por energía dispersiva de rayos $\mathrm{X}$ (EDX). Por último, se ha realizado una caracterización mecánica del material compuesto utilizando la técnica de nanoindentación.

\section{MÉTODO EXPERIMENTAL}

\subsection{Materias primas}

Thermal Ceramics ha suministrado preformas híbridas, compuestas por un $8 \%$ en volumen de fibras cortas (SAFFIL grado RF, ICI plc.) y un $16 \%$ en volumen de partículas (Microgrit WCA-25), en forma de discos de $330 \mathrm{~mm}$ de diámetro y $25 \mathrm{~mm}$ de espesor. La tabla I muestra las propiedades de las materias primas usadas en las preforma híbridas.

Se han mecanizado muestras de $60 \times 40 \times 25 \mathrm{~mm}$ y se han infiltrado con la cantidad correspondiente de aleación Al-8Mg mediante el proceso PRIMEX ${ }^{\mathrm{TM}}$, detallado a continuación. La aleación Al-8Mg se ha preparado mezclando aluminio puro comercial y aleación de magnesio AZ91 (Mg-9Al-1Zn). Para ello, se ha fundido el aluminio en un crisol de $\mathrm{SiC}$ y, tras fundirse completamente, se ha añadido la aleación

Tabla I. Principales características de las materias primas usadas en las preformas híbridas

Table I. Main characteristics of the raw materials used in the hybrid preforms

\begin{tabular}{llrlll}
\hline \multicolumn{1}{c}{ Nombre } & \multicolumn{1}{c}{ Tipo } & \% peso & Composición & Densidad & Forma \\
\hline Saffil & Fibras & 29,5 & $96 \% \mathrm{Al}_{2} \mathrm{O}_{3} \mathrm{y}$ & $3,3\left(\mathrm{~g} / \mathrm{cm}^{3}\right)$ & $\begin{array}{l}\text { Redondas } \\
(3 \mu \mathrm{m} \varnothing)\end{array}$ \\
Microgrit WCA-25 & $\begin{array}{l}\text { alúmina } \\
\text { Partículas } \\
\text { alúmina }\end{array}$ & 70,5 & $99,5 \% \mathrm{Al}_{2} \mathrm{O}_{3}$ & $3,9\left(\mathrm{~g} / \mathrm{cm}^{3}\right)$ & $\begin{array}{l}\text { Placas } \\
\text { hexagonales }\end{array}$ \\
\hline
\end{tabular}


de magnesio. A la hora de preparar las aleaciones Al-Mg, se ha añadido un $10 \%$ de magnesio, en exceso, para compensar las pérdidas por evaporación. Por último, se ha colado la aleación $\mathrm{Al}-8 \mathrm{Mg}$ en un molde de grafito para obtener lingotes con un tamaño de $250 \times 120 \times 25 \mathrm{~mm}$.

\subsection{Proceso de infiltración}

La figura 1 muestra un esquema del montaje empleado, en la presente investigación, para la infiltración de las preformas. Entre la preforma y la aleación se coloca una mezcla formada por polvo de alúmina y un $5 \%$ de polvo de magnesio, como iniciador del proceso de infiltración.

Se introduce el montaje en el interior del horno que, previamente, ha sido evacuado y llenado de $\mathrm{N}_{2}$, para empezar el ciclo de calentamiento. El proceso tiene dos fases bien diferenciadas. En la primera fase, se calienta el horno hasta la temperatura de nitruración, de $580^{\circ} \mathrm{C}$, a una velocidad de $200^{\circ} \mathrm{C} / \mathrm{h}$. Se mantiene a la temperatura de nitruración durante 5 $h$, para dar tiempo a que reaccione el magnesio con el $\mathrm{N}_{2}$. A continuación, se aumenta a la temperatura de nitruración hasta alcanzar la temperatura de infiltración, de $800^{\circ} \mathrm{C}$, a una velocidad de $100^{\circ} \mathrm{C} / \mathrm{h}$, y se mantiene durante $8 \mathrm{~h}$. Todo el proceso se lleva a cabo con un flujo de $\mathrm{N}_{2}$ continuo.

\subsection{Caracterización microestructural y química del material compuesto}

La caracterización microestructural y química del material compuesto se realizó mediante SEM

Atmósfera de $\mathrm{N}_{2}$

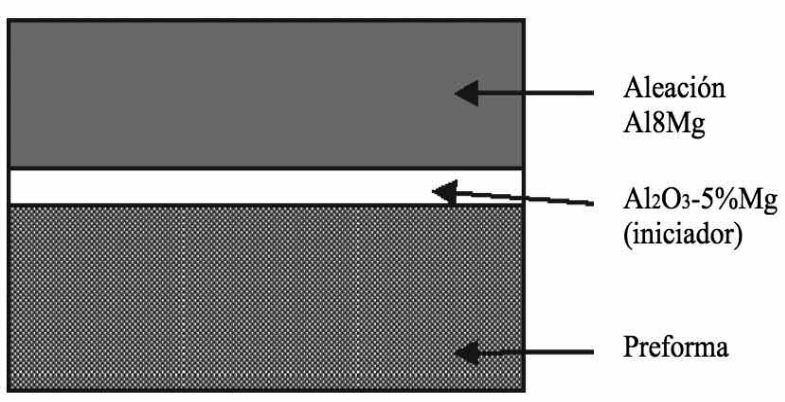

Figura 1. Ilustración esquemática del proceso de infiltración sin presión utilizado.

Figure 1. Schematic illustration of the used pressureless infiltration process.
(Hitachi SN 3400) y mediante análisis semicuantitativo por EDX (Bruker XFlash Detector 5010). La posible presencia de nitruro de aluminio se ha estudiado mediante la técnica de difracción de rayos $\mathrm{X}$ (Philips, PW3040/00 X'Pert MPD/MRD).

Para la caracterización mecánica del material compuesto se empleó un nanoindentador (Nanoindenter XP de MTS), aplicando cargas máximas de 10 y $20 \mathrm{mN}$, utilizando una punta de diamante con geometría Berkovich. Mediante estos ensayos se ha podido determinar el módulo de elasticidad $(E)$ y la dureza $(H)$ de las distintas regiones del material, a partir de las curvas de descarga y empleando el método de Oliver y Pharr ${ }^{[5]}$. Para poder determinar la influencia del refuerzo (tanto de fibras Saffil como partículas de alúmina) en las propiedades mecánicas de la matriz de $\mathrm{Al}-8 \mathrm{Mg}$ y la posible presencia de una intercara matriz-refuerzo de naturaleza mecánica distinta, se diseñaron matrices de nanoindentación en distintas zonas de la superficie del material. La localización de la huella de cada ensayo se ha realizado mediante SEM, asignando las propiedades mecánicas a cada zona de la microestructura del material y determinando la posible formación de fases entre las intercaras matriz-refuerzo con distinta naturaleza o comportamiento mecánico.

\section{RESULTADOS Y DISCUSIÓN}

\subsection{Caracterización microestructural}

La microestructura del material compuesto a bajos aumentos se presenta en la figura 2 a). Mediante el proceso de fabricación descrito se ha conseguido fabricar, por infiltración, un material compuesto de matriz de aluminio con un volumen de refuerzo del $16 \%$ en partículas de $\mathrm{Al}_{2} \mathrm{O}_{3}$ (señaladas en la figura 2 a)) y un $8 \%$ en fibras Saffil (indicado en la figura 2 b)) en direcciones paralela, fibra alargada, y perpendicular, sección circular, a la superficie estudiada).

Durante el proceso de fabricación se han formado dos tipos de precipitados intermetálicos: unos, cuya composición corresponde a $\mathrm{AlFeMg}$ (visibles como productos brillantes en la figura 2 a)), por contar con elementos de alto número atómico) y, otros, de composición AlSiMg, alrededor de las fibras de Saffil que presentan una morfología característica (Fig. 2 b)). No se ha apreciado, en análisis EDX, la presencia de nitrógeno en las intercaras, si bien esta técnica es poco sensible a este elemento, a bajas concentraciones.

Del análisis de las intercaras entre el refuerzo y la matriz (Fig. 3 a) y b)) se desprende que las partícu- 

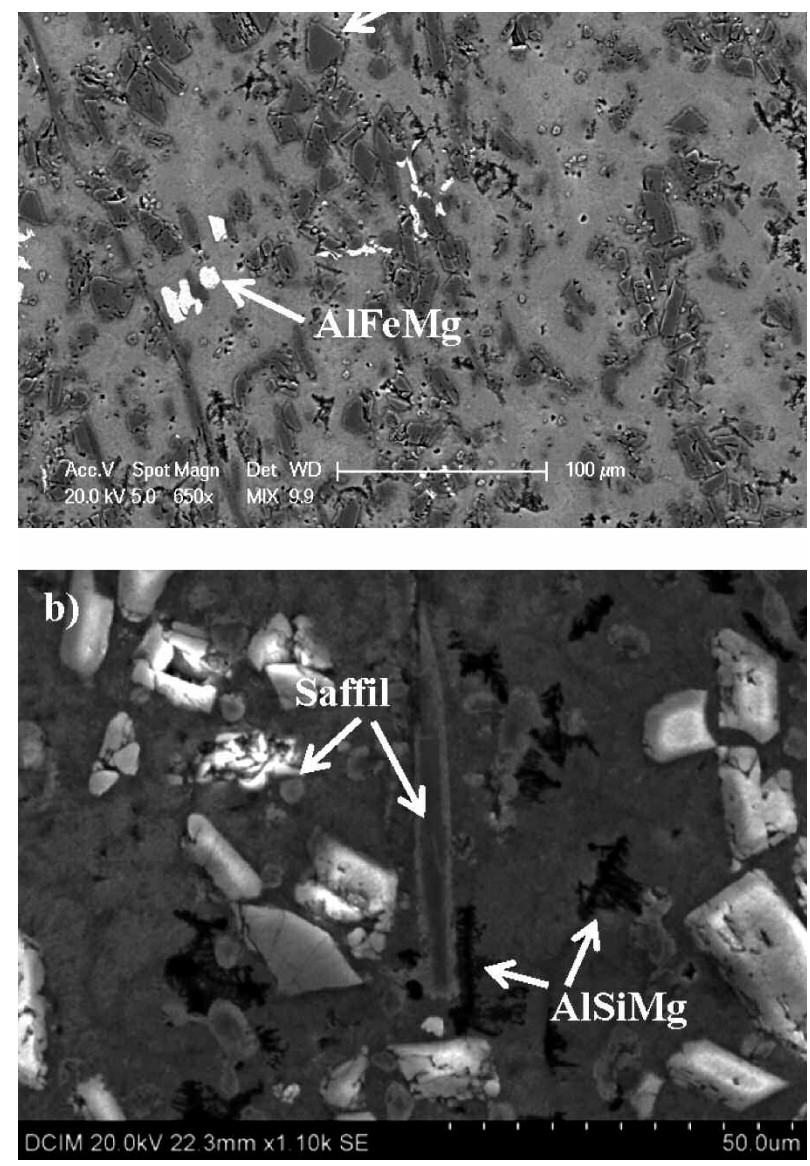

Figura 2. Micrografías SEM de la microestructura del material fabricado: a) vista general; b) detalle.

Figure 2. SEM micrography of the fabricated material microstructure: a) general view; b) detail.

las de alúmina no interaccionan con la matriz, si bien sus intercaras actúan favoreciendo la formación de los precipitados ricos en hierro en algunas zonas del material. Por otra parte, las fibras de Saffil sí reaccionan con su entorno; por tanto, la sílice de las fibras activa la reacción con el magnesio de la matriz, a través de la siguiente reacción:

$$
\mathrm{SiO}_{2}+2 \mathrm{Mg} \rightarrow 2 \mathrm{MgO}+\mathrm{Si}
$$

$\mathrm{El} \mathrm{MgO}$ es altamente reactivo con la alúmina y suele actuar según la reacción:

$$
\mathrm{Al}_{2} \mathrm{O}_{3}+\mathrm{MgO} \rightarrow \mathrm{MgAl}_{2} \mathrm{O}_{4}
$$

Por otra parte, no se ha observado silicio primario, por tanto debe haber reaccionado para formar los intermetálicos AlSiMg, alrededor de las fibras. Los ensayos de difracción de rayos X (Fig. 4) han
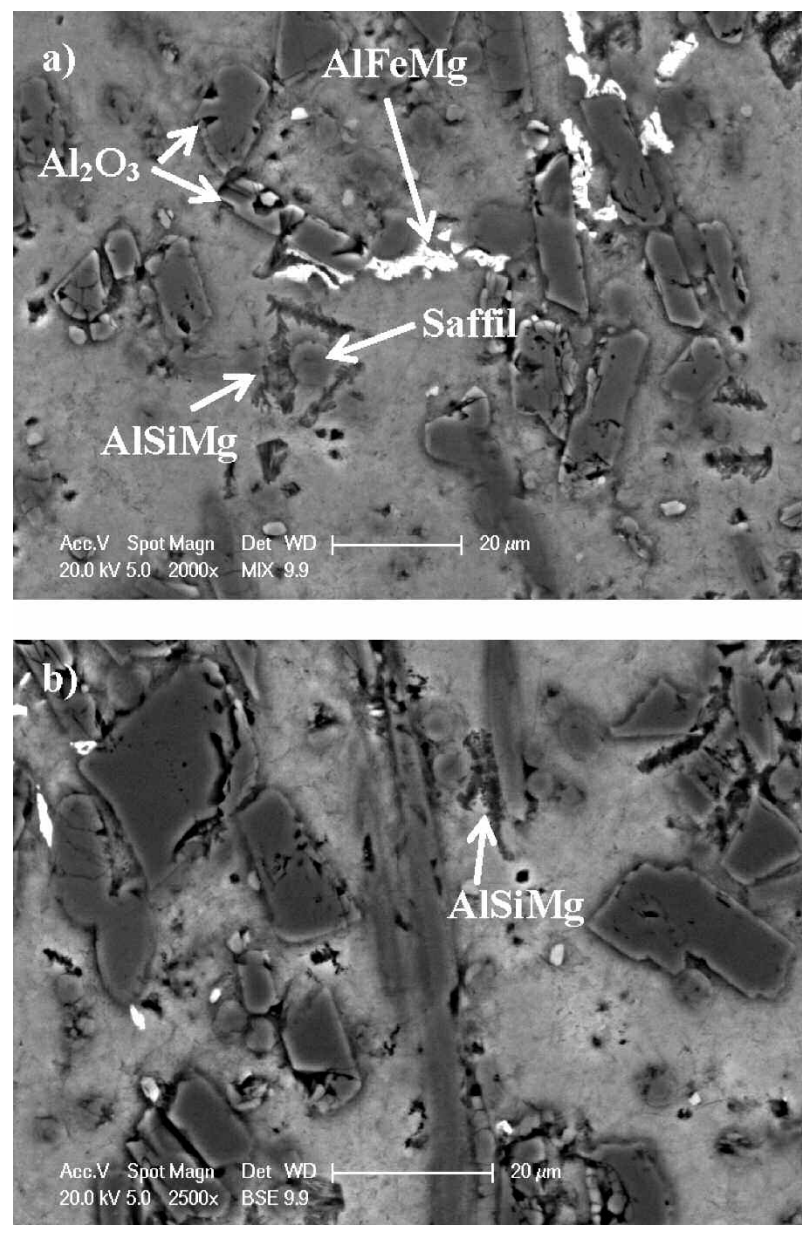

Figura 3. Estudio mediante SEM de las intercaras matriz-refuerzo: a) detalle de ubicación de AIFeMg; b) formación de AISiMg alrededor de fibras de Saffil.

Figure 3. Study by SEM of the matrixreinforcement interfaces: a) location detail of AlFeMg; b) formation of AISiMg around the Saffil fibres.

mostrado que en el material se encuentra $\mathrm{Al}, \mathrm{Al}_{2} \mathrm{O}_{3}$, $\mathrm{MgAl}_{2} \mathrm{O}_{4}$ y AlN. La observación de la espinela $\mathrm{MgAl}_{2} \mathrm{O}_{4}$ verifica la propuesta de reacción anterior y justifica la formación de productos en las intercaras de las fibras.

Por otra parte, la presencia de AlN es habitual en los procesos de infiltración sin presión asistidos con nitrógeno. En este proceso sobre fibras Saffil y partículas de alúmina, el AlN se encuentra en pequeñas cantidades. Aunque no ha sido observado en las zonas de la muestra estudiadas por SEM, su presencia sí ha sido detectada por EDX, en las zonas de precipitados de AlSiMg que rodean a las fibras de Saffil, a diferencia de lo que se suele observar cuando se emplea el proceso de infiltración sobre partículas de $\mathrm{SiC}$, en 


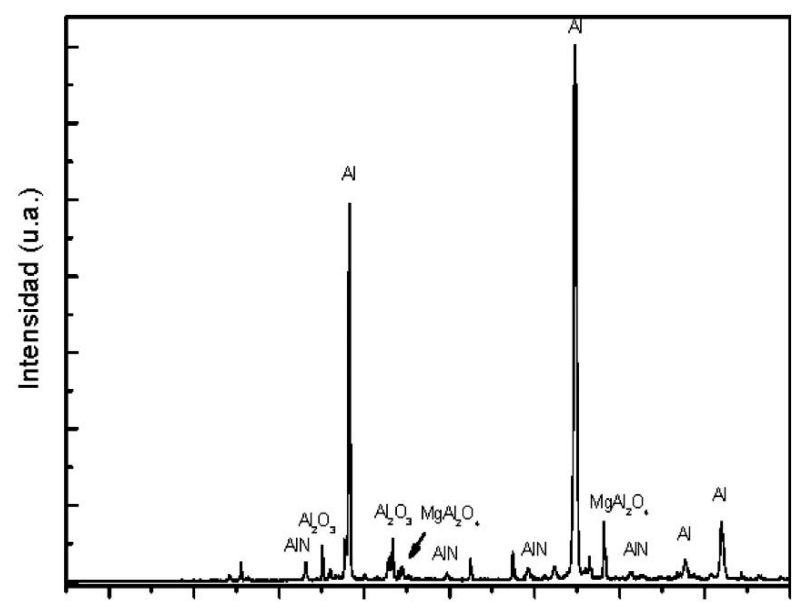

$10203040506070802 \theta$

Figura 4. Espectro de difracción de rayos $\mathrm{X}$ del material compuesto.

Figure 4. X-ray diffraction spectrum of the composite material.

el que la formación de nitruro de aluminio es más habitual en las intercaras de las partículas.

Finalmente, el material es compacto y solo se han observado poros en las zonas de acumulación de partículas y debido a su fractura. Esta aglomeración probablemente se haya producido durante el proceso de fabricación de la preforma y no durante el proceso de infiltración.

\subsection{Caracterización nanoindentación}

mecánica

por

En la figura 5 se puede apreciar una de las matrices de nanoindentación realizada para la caracterización mecánica de las distintas regiones del material compuesto; la circunferencia destaca una de las huellas residuales de un ensayo. Para cada indentación, se determina la dureza y, combinando estos valores con la localización en la micrografía, se determinan las propiedades mecánicas de las diferentes fases del material compuesto. Los valores medidos en las zonas se recogen en la tabla II.

Como cabía esperar, la matriz de aluminio es la región del material más blanda, los dos tipos de refuerzo utilizados poseen una dureza media muy superior a la de la matriz y los intermetálicos presentan una dureza intermedia.

En las zonas de intercara se ha observado que se produce una variación significativa de las propiedades mecánicas. La figura 6 a) muestra la diferencia de tamaño de la huella residual realizada sobre la matriz

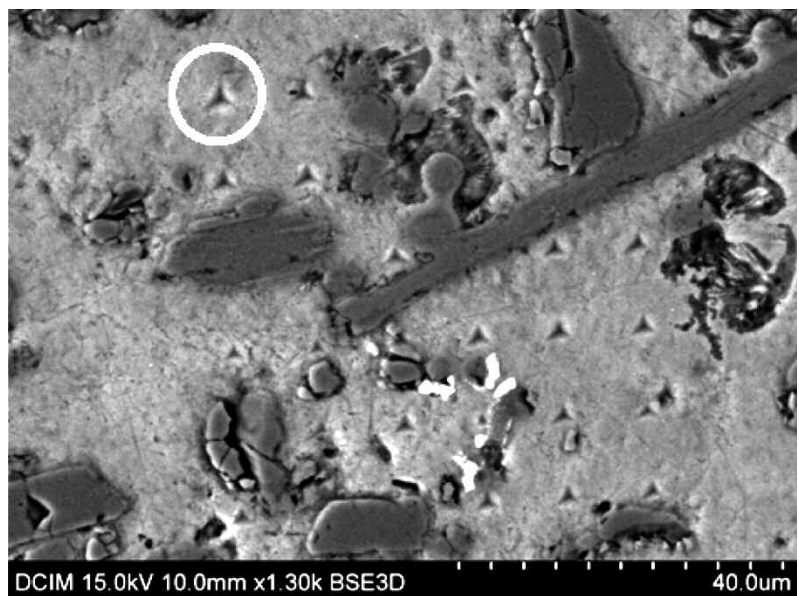

Figura 5. Micrografía SEM de una matriz de nanoindentaciones. La circunferencia muestra una de las huellas residuales marcada en la imagen.

Figure 5. SEM micrography of a nanoindentation matrix. The circumference shows one of the residual marks in the image.

Tabla II. Dureza media y desviación estándar medida en los ensayos de nanoindentación

Table II. Average hardness and standard deviation of the nanoindentation tests

\begin{tabular}{lc}
\hline \multicolumn{1}{c}{ Zona } & H (GPa) \\
\hline Matriz Al-8Mg & $1,71 \pm 0,28$ \\
Fibra Saffil & $9,5 \pm 1,2$ \\
Intercara matriz/fibra & $3,53 \pm 0,79$ \\
Partícula de $\mathrm{Al}_{2} \mathrm{O}_{3}$ & $12,4 \pm 4,2$ \\
Matriz cercana a partícula de $\mathrm{Al}_{2} \mathrm{O}_{3}$ & $2,93 \pm 0,74$ \\
Intermetálico AlSiMg & $3,6 \pm 2,1$
\end{tabular}

(indentación 1) y sobre la intercara (indentación 2), que demuestra la diferencia de dureza de estas dos zonas. Las curvas de carga - desplazamiento que corresponden a estas huellas (Fig. 6 b)) evidencian esta diferencia desde los primeros instantes de la indentación (señalado como A), mostrando que se trata de una zona más rígida y dura que la matriz. Posteriormente, se produce el contacto con la fibra que se manifiesta como un cambio de pendiente en la curva de carga (zona B). Finalmente, durante la curva de descarga se observa una discontinuidad (zona C), que se aprecia en la presencia de dos pendientes de recuperación elástica distintas. Por tanto, la dureza medida en esta zona se debe a la participación en el 


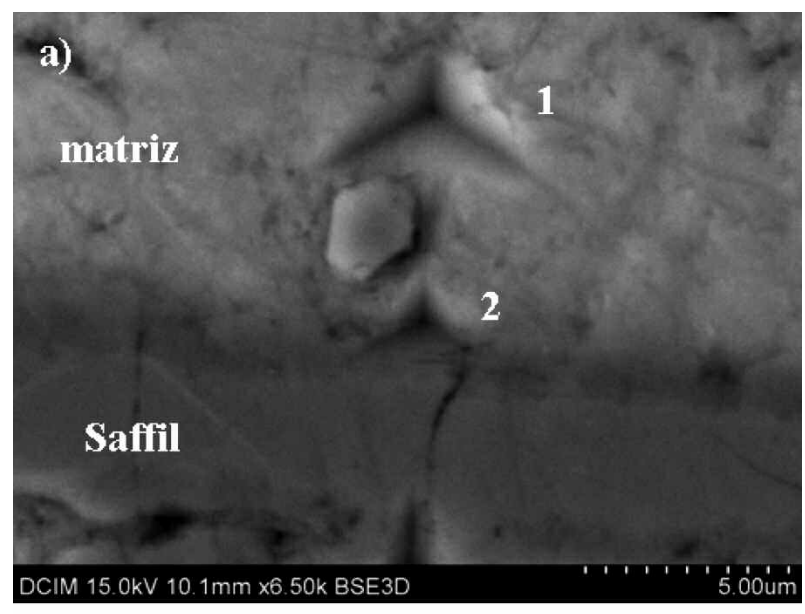

b)

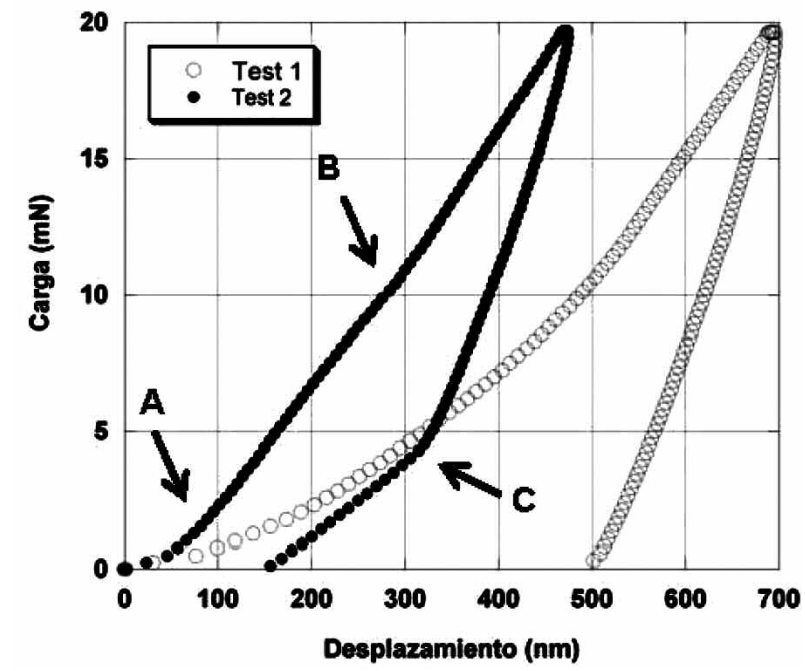

Figura 6. a) Micrografía SEM de huellas residuales de ensayos de nanoindentación en el entorno de una fibra Saffil; b) Gráficas cargadesplazamiento obtenidas en los ensayos de nanoindentación anteriores.

Figure 6. a) SEM micrography of the residual marks left by the nanoindentation tests around a Saffil fibre; b) load-displacement curves obtained in the nanoindentation tests.

ensayo de dos zonas del material compuesto, con dureza muy distinta (intercara y fibra) dando, como consecuencia, unos valores de dureza intermedios entre los observados anteriormente.

En la figura 7 se representa la dureza medida en función de la distancia a las fibras. Se observa que, independientemente del comportamiento de las intercaras, la presencia de las fibras de Saffil tiene una influencia muy escasa en el aumento de dureza de la matriz de $\mathrm{Al}-8 \mathrm{Mg}$ que la rodea a distancias superiores a $3 \mu \mathrm{m}$. A distancias inferiores, sí se observa un

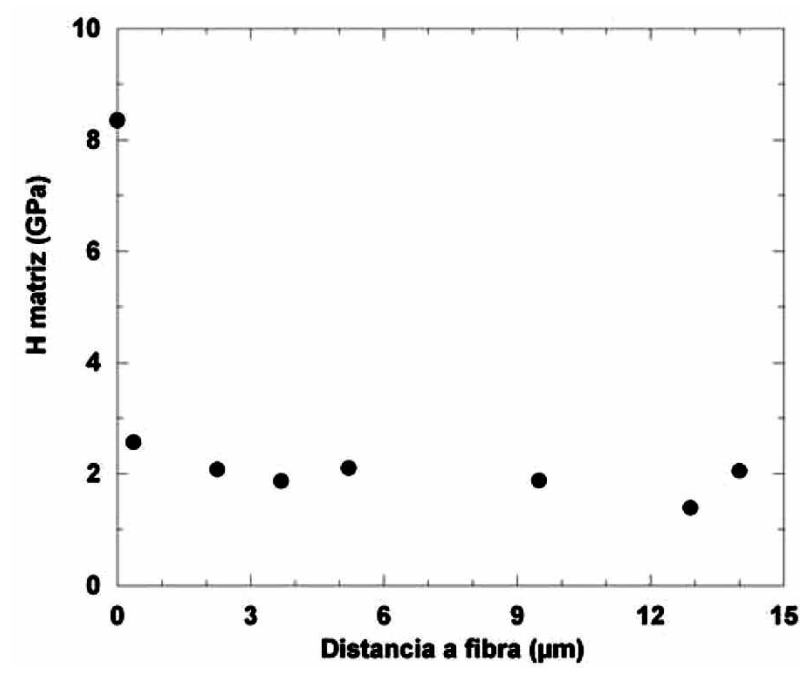

Figura 7. Variación de la dureza de la matriz de Al-8Mg en función de la distancia a fibras Saffil.

Figure 7. Variation of the Al-8Mg matrix hardness according to the distance to the Saffil fibre.

ligero endurecimiento que, a una distancia de $0,4 \mu \mathrm{m}$, asciende a 2,56 GPa, mientras que, a 2,25 $\mu \mathrm{m}$, la matriz posee, prácticamente, la misma dureza $(2,07$ $\mathrm{GPa})$ que en la zona libre de la influencia de la fibra (1,71 GPa).

La misma situación se manifiesta en el caso de las partículas de refuerzo, ya que la matriz que rodea a dichas partículas aumenta su dureza (Tabla II) pero, a pocas micras de distancia, la matriz recupera su dureza inicial. A diferencia de lo observado en el caso de las fibras, en este caso, no se ha observado la existencia de ninguna zona de reacción y el endurecimiento observado sólo puede deberse a la presencia de variaciones en la concentración de la matriz o al fenómeno de limitación de la deformación por la presencia de la partículas de alúmina. Desde cualquiera de estas dos perspectivas, se aprecia que se tiene una buena unión entre el refuerzo y la matriz.

A partir de los ensayos de nanoindentación, también, se puede determinar el módulo elástico, pero a diferencia de la dureza, el módulo elástico se determina, sólo, durante las primeras etapas de descarga del nanoindentador. Por tanto, los valores obtenidos están determinados por las zonas más externas de la huella de indentación, lo que implica que los valores en las proximidades de los refuerzos sobrevaloren la rigidez y no puedan considerarse como válidos de la rigidez de las intercaras estudiadas.

Finalmente, como cabía esperar por su ductilidad, no se ha observado la formación de grietas en los vértices de las huellas de indentación realizadas en la matriz. Por otra parte, es importante considerar que 
estas fracturas tampoco se han observado en las zonas más frágiles como son las intercaras y la propia zona de reacción entre fibras y matriz. Estos comportamientos, al igual que los observados anteriormente, son indicativos de que se cuenta con una buena intercara que puede limitar los problemas de fragilidad que surgen en muchos materiales compuestos.

\section{CONCLUSIONES}

- De la caracterización microestructural y química se concluye que, durante el proceso de fabricación, se han formado dos tipos de precipitados intermetálicos: AlFeMg y AlSiMg.

- Del análisis de las intercaras entre el refuerzo y la matriz se desprende que las partículas de alúmina no interaccionan con la matriz, si bien sus intercaras actúan favoreciendo la formación de los precipitados ricos en hierro, AlFeMg.

- Las fibras Saffil sí reaccionan con su entorno. Presumiblemente, la sílice activa la reacción formando $\mathrm{MgO}$, que es altamente reactivo con la alúmina y que ha acabado produciendo la espinela $\mathrm{MgAl}_{2} \mathrm{O}_{4}$. $\mathrm{Al}$ no observarse silicio prima- rio se deduce que debe haber reaccionado para formar el intermetálico AlSiMg.

- De la caracterización mecánica por nanoindentación en la intercara se concluye que se tiene una buena unión entre los refuerzos y la matriz.

\section{Agradecimientos}

Este trabajo ha sido financiado por el proyecto MAT2009-09845-C02-02.

\section{REFERENCIAS}

[1] B.S. Rao y V. Jayaram, Acta Mater. 49 (2001) 2.373-2.385.

[2] J. Liu, Z. Zheng, J. Wang, Y. Wu, W. Tang y J. Lü, J. Alloys Comp. 465 (2008) 239-243.

[3] R. Tavangar, L. Weber y A. Mortensen, Mater. Sci. Eng. A 395 (2005) 27-34.

[4] H. Chang, R.L. Higginson, J.G.P. Binner, J. Microsc. 233 (2009) 132-139.

[5] W.C. Oliver y G.M. Pharr, J. Mater. Res. 7 (1992) 1.564-1.583. 